\title{
Upregulation of P21-Activated Kinase 1 (PAK1)/CREB Axis in Squamous Non-Small Cell Lung Carcinoma
}

\author{
Jae Heun Chung ${ }^{a}$ Dae Hyun Kim ${ }^{d}$ Yun Seong Kim ${ }^{a}$ Bong Soo Son ${ }^{b}$ \\ Dohyung Kim $^{b}$ Chungsu Hwang ${ }^{c}$ Donghoon Shin ${ }^{c}$ Sang Gyun Noh ${ }^{d}$ \\ Jun Hee Hane Dae Kyung Kim ${ }^{f}$ Jae Ho Kim ${ }^{f}$ Ja Seok Koog \\ Hae Young Chung ${ }^{d}$ Seong Hoon Yoon ${ }^{a}$
}

\begin{abstract}
${ }^{a}$ Department of Internal Medicine, Pusan National University Yangsan Hospital, Yangsan, bepartment of Thoracic and Cardiovascular Surgery, Pusan National University Yangsan Hospital, Yangsan, 'Department of Pathology, Pusan National University Yangsan Hospital, Yangsan, dMolecular Inflammation Research Center for Aging intervention (MRCA), College of Pharmacy, Pusan National University, Busan, eDepartment of Statistics, Hallym University, Chuncheon, Kangwon, fDepartment of Physiology, School of Medicine, Pusan National University, Yangsan, Korea, 'Section of Medical Oncology, Department of Internal Medicine, Yale Comprehensive Cancer Center, Yale School of Medicine, New Haven, USA
\end{abstract}

\section{Key Words}

p21-activated Ser/Thr kinase 1 - Cyclic AMP response element-binding protein • Squamous cell lung cancer $\cdot$ NSCLC

\begin{abstract}
Background/Aims: p21-activated Ser/Thr kinase 1 (PAK1) is essential for the genesis and development of many cancers. The purpose of this study was to investigate the role of the PAK1-cyclic AMP response element-binding (CREB) axis in non-small cell lung cancer (NSCLC) tumorigenesis and its related mechanisms. Methods: Western blot assay and immunohistochemical staining were employed to investigate the PAK1 and CREB expression in the tissue microarray of human squamous NSCLC. Co-immunoprecipitation and immunofluorescence confocal assays were performed to determine the link between PAK1 and CREB. NSCLC xenograft models were used to study oncogenic function of PAK1 in vivo. Results: We observed that PAK1 and CREB expression levels were significantly elevated in human squamous NSCLC-tissue specimens, compared with those in adjacent normal bronchial or bronchiolar epithelial-tissue specimens, as well as their phosphorylated forms, based on western blotting. We showed in vitro that PAK1 knockdown by small-interfering RNA (siRNA) blocked CREB phosphorylation, whereas plasmid-based PAK1 overexpression resulted in CREB phosphorylation at Ser133, based on western blotting. In addition, PAK1 interacted

JH. Chung and DH. Kim contributed equally to this work.

\begin{tabular}{ll}
\hline Hae Young Chung & College of Pharmacy, Pusan National University, Geumjeong-Gu, Busan 46241; \\
and Seong Hoon Yoon & Pusan National University Yangsan Hospital, Yangsan-si, Gyeongsangnam-do 50612 (Korea) \\
& E-Mail hyjung@pusan.ac.kr; drysh79@gmail.com
\end{tabular}
\end{abstract}


with CREB in co-immunoprecipitation assays. Additionally, our in vitro findings detected by flow cytometry revealed that PAK1 silencing attenuated cell cycle progression, inducing apoptosis. Inhibition of PAK1 expression reduced tumor sizes and masses by modulating CREB expression and activation in xenograft models. Conclusion: These results suggest a novel mechanism whereby the PAK1-CREB axis drives carcinogenesis of squamous-cell carcinomas, and have important implications in the development of targeted therapeutics for squamouscell lung cancer.

(C) 2018 The Author(s)

Published by S. Karger AG, Basel

\section{Introduction}

The introduction of targeted therapy offers new hope to lung cancer patients by precisely directing treatment to signaling pathways related to carcinogenesis and tumor growth. Therefore, the discovery of new molecular targets for treating non-small cell lung cancer (NSCLC) can improve the control of untreated lung cancer.

Recent data have shown that p21-activated kinase (PAK) contributes to a wide range of cellular processes, including actin cytoskeleton reorganization, cell survival, and cell proliferation [1]. To date, six PAK family members have been identified in mammalian cells and classified into groups I (PAK1-3) and II (PAK4-6), based on their structural and functional similarities [2]. PAK1 is involved in the tumorigenesis of several types of cancer. For example, downregulating PAK1 expression with a short-hairpin RNA or treatment with the selective PAK1 inhibitor FRAX597 reduced pancreatic cancer cell growth and survival [3]. Moreover, PAK1 expression is upregulated in prostate cancer, and treatment with the mTOR inhibitor rapamycin slowed cancer cell proliferation by inhibiting PAK1 expression [4]. Furthermore, in human breast cancer cells, PAK1 can mediate cell proliferation and tumor growth, with prolactin treatment triggering PAK1-mediated estrogen receptor- $\alpha$ phosphorylation [5].

Several lines of evidence show that PAK1 is also involved in the treatment process of lung cancers. During radiotherapy, PAK1 overexpression induced by radiation in NSCLC cells correlates with the epithelial-mesenchymal transition through the regulation of epithelialand mesenchymal-cell markers, such as vimentin and N-cadherin levels [6]. Wu et al. [7] reported that the positive feedback loop associated with PAK1 and its related signaling cascades persistently activates the phosphoinositide 3-kinase/AKT-signaling pathway, resulting in an unfavorable response to targeted therapy through tyrosine-kinase inhibitors in patients with lung adenocarcinoma. PAK1 also correlates with resistance against cisplatin, which represents the most prevalent conventional chemotherapy in patients with NSCLC, via $\beta$-catenin-mediated stemness [8]. A recent report of particular interest revealed that PAK1 is required for NSCLC proliferation [9], showing that after PAK1 inhibition, cells showed increased expression of the cyclin-dependent kinase (CDK) inhibitor p27Kip1 and its accumulation in the G1 phase. Inhibition of PAK1 expression also significantly impaired tumor growth in a lung cancer xenograft model, supporting the role of PAK1 as a driver of tumor proliferation in NSCLC.

The cyclic AMP response element-binding protein (CREB) was shown to play a role in cell survival, proliferation, and cell cycle progression [10-12]. CREB overexpression results in significantly decreased survival duration in patients with NSCLC [13], whereas CREB knockdown induces cell cycle arrest and apoptosis in NSCLC cells [14]. Recent data showed that PAK4, a group II PAK, regulates prostate tumorigenesis and differentiation in a CREB-dependent manner [10]. However, it remains unclear whether PAK1 is associated with NSCLC by CREB phosphorylation. Based on these studies, we tested the hypothesis that PAK1 modulates CREB phosphorylation, which might play an important role in lung carcinogenesis as a regulatory factor in NSCLC. 


\section{Cellular Physiology Cell Physiol Biochem 2018;50:304-316

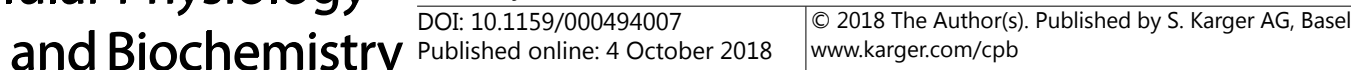

Chung et al.: PAK1/CREB Axis in Squamous NSCLC

\section{Materials and Methods}

\section{Materials}

All chemical reagents, except when specified, were obtained from Sigma-Aldrich (St. Louis, MO, USA). Antibodies specific for B-cell lymphoma 2 (Bcl-2), Bcl-2-associated X protein (Bax), PARP, caspase-3, and caspase-9 were purchased from Santa Cruz Biotechnology (Dallas, TX, USA). Antibodies specific for PAK1 (\#2602; Cell Signaling Technology, Danvers, MA, USA), phopsphorylated-PAK1 (p-PAK1; ab-75599; Abcam, Cambridge, UK), CREB (ab-109596; Abcam), p-CREB (sc-101663; Santa Cruz Biotechnology), and E2F8 (ab-109596; Abcam) were obtained from the indicated manufacturers. PAK1 (719300) and CREB (MA1083) antibodies (Invitrogen, Grand Island, NY, USA) were used for immunohistochemistry and immunofluorescence confocal assays. All other materials were obtained in the purest available grade.

\section{Cell culture}

Authenticated human squamous carcinoma (H1703) cells were acquired from the American Type Culture Collection (ATCC; http://www.atcc.org/) and maintained at early passages (no more than six months after receipt from the ATCC). Cells were cultured in RPMI-1640 medium (Invitrogen) supplemented with $10 \%(\mathrm{v} / \mathrm{v}$ ) heat-inactivated FBS (Sigma-Aldrich), $100 \mathrm{U} / \mathrm{mL}$ penicillin G sodium, and $100 \mu \mathrm{g} / \mathrm{mL}$ streptomycin sulfate (Invitrogen). Cells were cultured at $37^{\circ} \mathrm{C}$ in a humidified atmosphere of $95 \%$ air and $5 \% \mathrm{CO}_{2}$.

Lung-tissue homogenates and western blot analysis

Tissue pieces $(0.1 \mathrm{~g})$ were transferred to separate 2-mL centrifuge tubes containing stainless steel beads. CETi lysis buffer (Translab, Daejeon, Korea) was immediately added and the tissues were subjected to oscillation using a TissueLyser II machine (Qiagen, Hilden, Germany) at $30 \mathrm{~Hz}$ for $3 \mathrm{~min}$. Each lysate was incubated for $15 \mathrm{~min}$ on ice, followed by centrifugation at $12,000 \times g$ for $10 \mathrm{~min}$ at $4{ }^{\circ} \mathrm{C}$. The supernatants were retained, and their protein concentrations were measured using a bicinchoninic acid assay (Thermo Fisher Scientific, Waltham, MA, USA). The samples were prepared in gel-loading buffer $(0.125 \mathrm{M}$ Tris- $\mathrm{HCl}$ [pH 6.8], 4\% SDS, 10\% 2-mercaptoethanol, and $0.2 \%$ bromophenol blue) at a 1:1 volumetric ratio and boiled for $5 \mathrm{~min}$. Western blot analysis was performed as described previously [15].

\section{Plasmid constructs and transfection}

The PAK1-overexpression plasmid was a generous gift from Dr. Chernoff (Fox Chase Cancer Center, University of Pennsylvania, Philadelphia, PA, USA). Three PAK1 siRNAs were obtained from a commercial source (IDT, Coralville, Iowa, USA). Transfection was performed using Lipofectamine 2000 reagent (Invitrogen), and cells were treated with scrambled or PAK1 siRNA-Lipofectamine complexes (25 nM) in Opti-MEM reagent (Invitrogen) in the absence of serum. After a 4 -h incubation at $37^{\circ} \mathrm{C}$, the transfection medium was replaced with fresh medium and the cells were incubated for another $48 \mathrm{~h}$.

\section{NSCLC-tissue specimens and Tissue microarray (TMA) construction}

Sixteen frozen tumor-tissue specimens (eight adenocarcinoma and eight squamous-cell carcinoma) and adjacent normal lung-tissue specimens, surgically resected from patients who underwent lobectomies for primary NSCLC, were provided by the Biobank of Pusan National University Hospital, a member of the Korea Biobank Network, and the Pusan National University Yangsan Hospital. All tumors were histologically examined and classified using the 2015 World Health Organization International Classification of Lung Tumors.

Specimens of tumor and adjacent normal lung tissues, obtained from 64 patients with surgically resected NSCLC (32 adenocarcinoma and 32 squamous-cell carcinoma), were assessed immunohistochemically for PAK1 and CREB expression in whole paraffin-embedded sections. Tumor TMAs were constructed using three tissue cores (1-mm diameter) per tumor to obtain tissue from central, intermediate, and peripheral tumor areas after histologic examination of 60 squamous NSCLC specimens in the Pathology Department of Pusan National University Yangsan Hospital. This protocol was approved by the Institutional Review Board protocol (04-2017-004) of Pusan National University Yangsan Hospital. 


\section{Cellular Physiology Cell Physiol Biochem 2018;50:304-316 \begin{tabular}{ll|l} 
and Biochemistry Publisned onIIne: 4 Uctober 2018 & $\begin{array}{l}\text { (c) } 2018 \text { The Author(s). Published by S. Karger AG, Basel } \\
\text { www.karger.com/cpb }\end{array}$
\end{tabular}}

Chung et al.: PAK1/CREB Axis in Squamous NSCLC

\section{Immunohistochemistry}

Immunohistochemical analysis of NSCLC- and adjacent normal-tissue specimens, and of the TMA NSCLCtissue specimens, was performed using anti-PAK1 and anti-p-PAK1 antibodies according to manufacturer's instructions. Immunostaining was visualized using the Cellsens standard from Olympus Life Sciences (DP21; Tokyo, Japan). Distinct nuclear immunostaining for antibodies was quantified by pathologists under a light microscope (Nikon Eclipse Ts2; Tokyo, Japan). Following tissue staining, the staining intensity in each slide was ranked on a scale from 0 to $3+$, with 0 indicating no staining, $1+$ indicating weak staining, 2+ indicating moderate staining, and $3+$ indicating strong staining.

\section{Immunofluorescence confocal assays}

For immunofluorescence staining, cells were fixed for 10 min in PBS containing 4\% paraformaldehyde, washed twice with PBS, and blocked in 5\% bovine serum albumin for $60 \mathrm{~min}$; all procedures were performed at room temperature. The fixed specimens were incubated with primary antibodies for $4 \mathrm{~h}$, followed by incubation with secondary antibodies for $1 \mathrm{~h}$. Primary antibodies (1:200) were detected by Alexa Fluor 488 and Alexa Fluor 568 conjugated secondary antibodies (1:1000) (Life Technologies). The specimens were finally washed and mounted in Vectashield medium (Vector Laboratories, Burlingame, CA) with 4',6-diamidino-2-phenylindole (DAPI) for visualization of nuclei. The stained sections were visualized using laser scanning confocal microscopy (Olympus FluoView FV1000).

\section{Apoptosis assay}

H1703 lung cancer cells were collected in tubes $48 \mathrm{~h}$ subsequent to transfection with siRNA and washed twice with cold PBS. Annexin V and PI staining were performed at room temperature for 20 min in the dark using the Annexin V-FITC Apoptosis Detection Kit (BD Biosciences, Inc., San Jose, CA), according to the manufacturer's instructions. Within $1 \mathrm{~h}$ of staining, the cells underwent flow cytometry, which was performed using a FACSCanto II flow cytometer (BD Biosciences). Viable cells were annexin V-/PI-, early apoptotic cells were annexin+/PI-, and late apoptotic/dead cells were annexin+/PI+.

\section{Cell cycle analysis}

H1703 lung cancer cells were harvested 48 h subsequent to transfection with siRNA, fixed in PBS containing 4\% paraformaldehyde overnight, washed twice with PBS, stained by PI ( $50 \mu \mathrm{g} / \mathrm{ml})$, and treated with RNase $(50 \mu \mathrm{g} / \mathrm{ml})$ in the dark at $37^{\circ} \mathrm{C}$ for $60 \mathrm{~min}$. Cell cycle stage analysis was performed by flow cytometry (FACSCantoII).

Terminal deoxynucleotidyl transferase dUTP nick-end labeling (TUNEL) assays

TUNEL assays were performed using an ApopTag Fluorescein Direct In Situ Apoptosis Detection Kit (Millipore, Billerica, MA, USA). Lung cancer cells were transfected with siRNAs for $48 \mathrm{~h}$, followed by fixation with 1\% paraformaldehyde in PBS ( $\mathrm{pH} 7.4$ ). Cells were washed with PBS, transferred to the equilibration buffer supplied in the kit, and incubated with the TdT enzyme in a humidified chamber at $37{ }^{\circ} \mathrm{C}$ for $1 \mathrm{~h}$. The cells were the incubated in working strength stop/wash buffer for $10 \mathrm{~min}$ and mounted with ProLong Gold Antifade reagent with DAPI (Invitrogen).

\section{Immunoprecipitation}

Immunoprecipitation experiments were performed by harvesting cells transfected with plasmids and preparing cell lysates in immunoprecipitation lysis buffer $(20 \mathrm{mmol} / \mathrm{L} \mathrm{Tris}-\mathrm{Cl}$ [pH 7.5], $150 \mathrm{mmol} / \mathrm{L} \mathrm{NaCl}$, $10 \%$ glycerol, and $1 \%$ Triton X-100). Cell extracts $(1.5 \mathrm{mg}$ ) were incubated with $40 \mathrm{~mL}$ of anti-agarose affinity gel (Millipore). After extensive washing with immunoprecipitation lysis buffer, the immunoprecipitated proteins were analyzed by immunoblotting, according to the manufacturer's instructions.

\section{In vivo studies}

All animal protocols were approved by the Institutional Animal Care and Use Committee at Pusan National University and conformed to guidelines for the care and maintenance of laboratory animals. Female BALB/c nude mice were obtained from OrientBio (Kyunggi, Korea) and used for experiments after they were 5-6 weeks old. H1703 cells were pretreated with $25 \mathrm{nM}$ of control siRNA or PAK1 siRNA for 48h, 


\section{Cellular Physiology Cell Physiol Biochem 2018;50:304-316

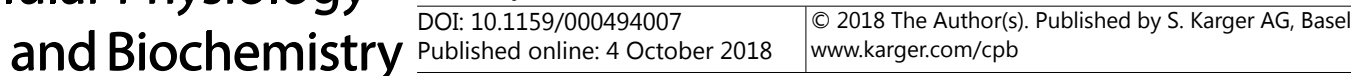 \\ Chung et al.: PAK1/CREB Axis in Squamous NSCLC}

followed by transplantation $\left(6 \times 10^{5}\right.$ cells/flank xenograft; $\left.n=10\right)$ into both right and left dorsal flanks of mice. Mice were sacrificed at the end of the study by being placed in a $\mathrm{CO}_{2}$ chamber.

\section{Statistical analysis}

The baseline characteristics of the data generated in this study were described in terms of the mean \pm SD, and Student's $t$ tests were performed to evaluate the significances of the differences between groups. Pearson's correlation coefficients were calculated to determine correlations among the variables. Differences in survival times (days) between groups were evaluated using Kaplan-Meier plots, and log-rank tests were performed to test the significances of the differences. Univariate Cox regression was also performed to determine which risk factors were significant, as well as their hazard ratios (HRs). P $<0.05$ was considered statistically significant.

\section{Results}

Associations between PAK1 and p-PAK1 levels with squamous-cell carcinomas, according to patient demographics and clinicopathological characteristics

PAK1 and p-PAK1 levels were also analyzed with tissue microarrays containing 60 squamous-cell carcinoma specimens. Our results showed that 40 of $60(67 \%)$ and 53 of $60(88 \%)$ squamous-cell NSCLC samples were positive for PAK1 and p-PAK1, respectively, and that $20 \%$ and $40 \%$ of all cases showed moderate (2+) or strong (3+) staining intensity for PAK1 and p-PAK1, respectively (mean \pm SD: $0.894 \pm 0.105$ for PAK1; and $1.298 \pm 0.096$ for p-PAK1; Table 1). The correlations between the clinical characteristics of patients with squamous cell lung cancer and PAK1 and p-PAK1 expression are summarized in Table 1. Furthermore, we also used univariate Cox proportional hazards-regression models to determine the effects of covariates on overall survival duration, showing that $\mathrm{N}$ classification and tumor-node-metastasis stage III significantly affected overall survival (Table 2). Representative images of PAK1 and p-PAK1 staining in the TMA specimens are shown in Fig. 1A. The Kaplan-Meier survival curves shown in Fig. 1B indicate that PAK1 and p-PAK1 levels correlated with survival rates; however, these levels were not significantly associated with decreased overall survival duration in patients with $\operatorname{NSCLC~}(\mathrm{P}=0.17, \mathrm{HR}=2.09$; and $\mathrm{P}=0.13$, $\mathrm{HR}=2.69$, respectively).

Table 1. Clinicopathological characteristics in tumor TMA specimens. A, Association of PAK1-immunostaining scores with clinicopathological characteristics in tumor TMA specimens

\begin{tabular}{|c|c|c|c|c|c|c|c|c|c|c|}
\hline \multirow{2}{*}{ Variables } & \multicolumn{4}{|c|}{ PAK1 Immunoscoring } & \multirow{2}{*}{$\mathrm{p}$ value } & \multicolumn{4}{|c|}{ p-PAK1 immunoscoring } & \multirow{2}{*}{$\mathrm{p}$ value } \\
\hline & $0(n=20)$ & $1(n=28)$ & $2(n=10)$ & $3(n=2)$ & & $0(n=7)$ & $1(n=29)$ & $2(n=21)$ & $3(n=3)$ & \\
\hline Mean age & 65 & 66 & 65 & 69 & 0.074 & 67 & 66 & 65 & 64 & 0.385 \\
\hline Sex & & & & & 0.861 & & & & & 0.664 \\
\hline Male & 17 & 27 & 9 & 2 & & 6 & 28 & 20 & 3 & \\
\hline Female & 1 & 1 & 1 & 0 & & 1 & 1 & 1 & 0 & \\
\hline $\mathrm{T}$ stage & & & & & 0.358 & & & & & 0.962 \\
\hline 1 & 9 & 6 & 5 & 0 & & 2 & 10 & 6 & 2 & \\
\hline 2 & 9 & 15 & 5 & 1 & & 4 & 15 & 10 & 1 & \\
\hline 3 & 2 & 5 & 0 & 1 & & 1 & 3 & 4 & 0 & \\
\hline 4 & 0 & 2 & 0 & 0 & & 0 & 1 & 1 & 0 & \\
\hline $\mathrm{N}$ stage & & & & & 0.925 & & & & & 0.769 \\
\hline 0 & 11 & 15 & 5 & 1 & & 2 & 15 & 12 & 3 & \\
\hline 1 & 5 & 6 & 3 & 1 & & 3 & 7 & 5 & 0 & \\
\hline 2 & 3 & 7 & 2 & 0 & & 2 & 6 & 4 & 0 & \\
\hline 3 & 1 & 0 & 0 & 0 & & 0 & 1 & 0 & 0 & \\
\hline M stage & & & & & 0.166 & & & & & 0.780 \\
\hline 0 & 20 & 28 & 9 & 2 & & 7 & 28 & 21 & 3 & \\
\hline 1 & 0 & 0 & 1 & 0 & & 0 & 1 & 0 & 0 & \\
\hline TNM stage & & & & & 0.388 & & & & & 0.518 \\
\hline I & 11 & 11 & 5 & 0 & & 2 & 12 & 10 & 3 & \\
\hline II & 4 & 9 & 3 & 2 & & 3 & 10 & 5 & 0 & \\
\hline III & 5 & 8 & 2 & 0 & & 2 & 7 & 6 & 0 & \\
\hline Smoking status & & & & & 0.750 & & & & & 0.664 \\
\hline Current & 5 & 11 & 5 & 1 & & 2 & 11 & 8 & 1 & \\
\hline Former & 15 & 16 & 5 & 1 & & 5 & 17 & 13 & 2 & \\
\hline Never & 0 & 1 & 0 & 0 & & 0 & 1 & 0 & 0 & \\
\hline
\end{tabular}




\section{\begin{tabular}{ll} 
Cellular Physiology & Cell Physiol Biochem 2018;50:304-316 \\
\hline DOI: 10.1159/000494007 & O 2018 The Author(s). Published by S. Karger AG, Basel
\end{tabular}

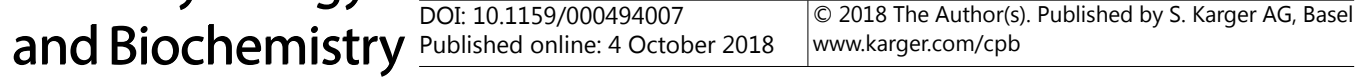 \\ Chung et al.: PAK1/CREB Axis in Squamous NSCLC}

PAK-1 and CREB expression were higher in frozen NSCLC tissue than in adjacent normal lung tissues

PAK-1 protein levels were elevated and more highly activated in the eight frozen NSCLCtissue specimens as compared with the PAK-1 levels observed in the adjacent normal-tissue specimens, according to western blot analysis, which revealed $\sim 3$ - to $\sim 4$-fold higher levels in NSCLC tissues than in normal tissues $(\mathrm{P}<0.01$ in adenocarcinoma tissues; $\mathrm{P}=0.015$ in squamous-cell carcinoma tissues; Fig. 2A). These eight NSCLC-tissues specimens also showed higher p-PAK1 levels when compared with those in normal tissues. Western blot analysis showed similar patterns for CREB and p-CREB levels, with $\sim 3$-fold higher levels in NSCLC tissues than in normal tissues $(\mathrm{P}<0.01$ in both adenocarcinoma and squamouscell carcinoma tissues; Fig. 2A). Immunohistochemical analysis of PAK1, p-PAK1, CREB, and p-CREB levels in 20 whole paraffin-embedded NSCLC-tissue specimens showed that the expression of PAK1, CREB, and their phosphorylated forms, was higher than that observed in adjacent normal tissues, in both adenocarcinoma and squamous-cell carcinoma tissues, based on western blot results (Fig. 2B). These results indicated that PAK1 and CREB were overexpressed and highly activated in NSCLC tissues. Additionally, nuclear localization of PAK1 and CREB was evident in a proportion of squamous-cell carcinoma tissues (Table 3).

\section{Role of PAK1 in proliferation of squamous-cell NSCLC}

cells

The biological role of PAK1 in squamous-cell carcinomas was further explored using gene set enrichment analysis (GSEA) based on mRNA-expression data derived from The Cancer Genome Atlas (TCGA), which indicated that high levels of PAK1 and CREB correlated significantly with cell cycle-associated gene signatures (Fig. 3A). We subsequently studied the effects of PAK1 siRNA-mediated knockdown in squamous-cell carcinomas to clarify the contribution of PAK1 to tumor-cell growth and survival. Examination of the contribution of PAK1 to cell cycle progression in H1703 cells (as determined by fluorescence-activated cell-sorting analysis) showed that PAK1 silencing stimulated increased cell cycle arrest at the G1/S phase in cells cultured with $25 \mathrm{nM}$ PAK1 siRNA stationary in the G1/S phase, as compared with control cells in the G1/S phase (Fig. 3B). Additionally, annexin-V-
Table 2. Estimation of overall survival (OS) durations related to each clinicopathological characteristic of NSCLC patients, according to TMA and univariate Cox proportional hazards regression models

\begin{tabular}{lcc}
\hline Variable & \multicolumn{2}{c}{ OS } \\
& HR & P value \\
\hline Age & 0.6 & 0.48 \\
Sex & 1.02 & 0.62 \\
Smoking & 0.74 & 0.58 \\
T stage & 1.92 & 0.31 \\
N stage & 3.52 & 0.03 \\
TNM stage & 3.58 & 0.04 \\
\hline
\end{tabular}

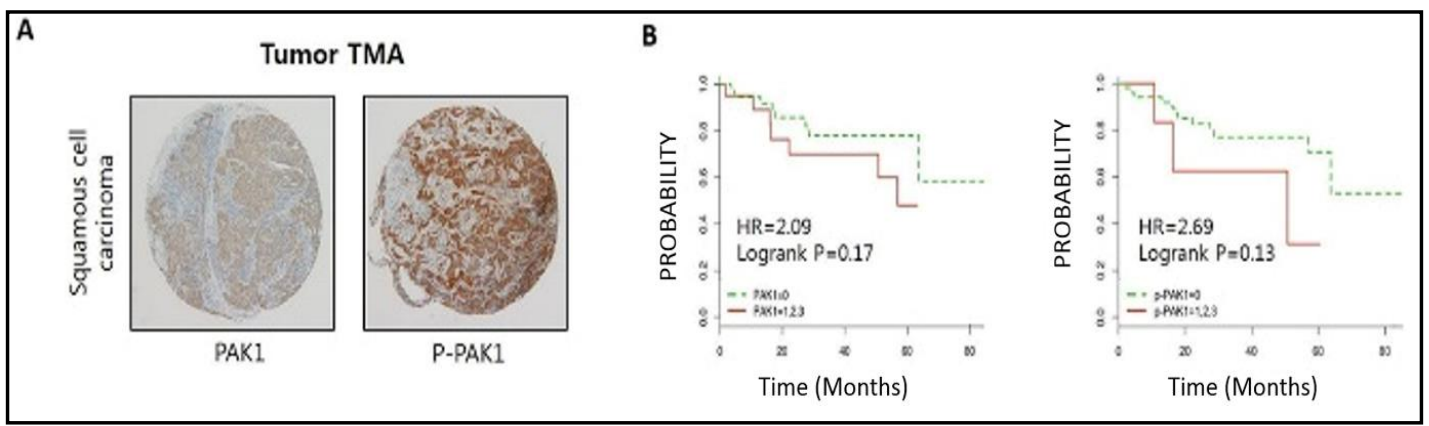

Fig. 1. PAK1 and p-PAK1 immunostaining of TMA specimens obtained from 60 patients with squamous-cell carcinoma. A, Representative images of PAK1 and p-PAK1 immunostaining of TMA squamous-cell carcinoma specimens. Magnification: 40X. B, Kaplan-Meier curves of overall survival durations, according to the PAK1 (left) and p-PAK1 (right) levels. The median overall survival duration was 56.9 months and 50.8 months (PAK1 and p-PAK1 = 1,2,3; relatively). The curves are characterized by the corresponding immunostaining scores. 


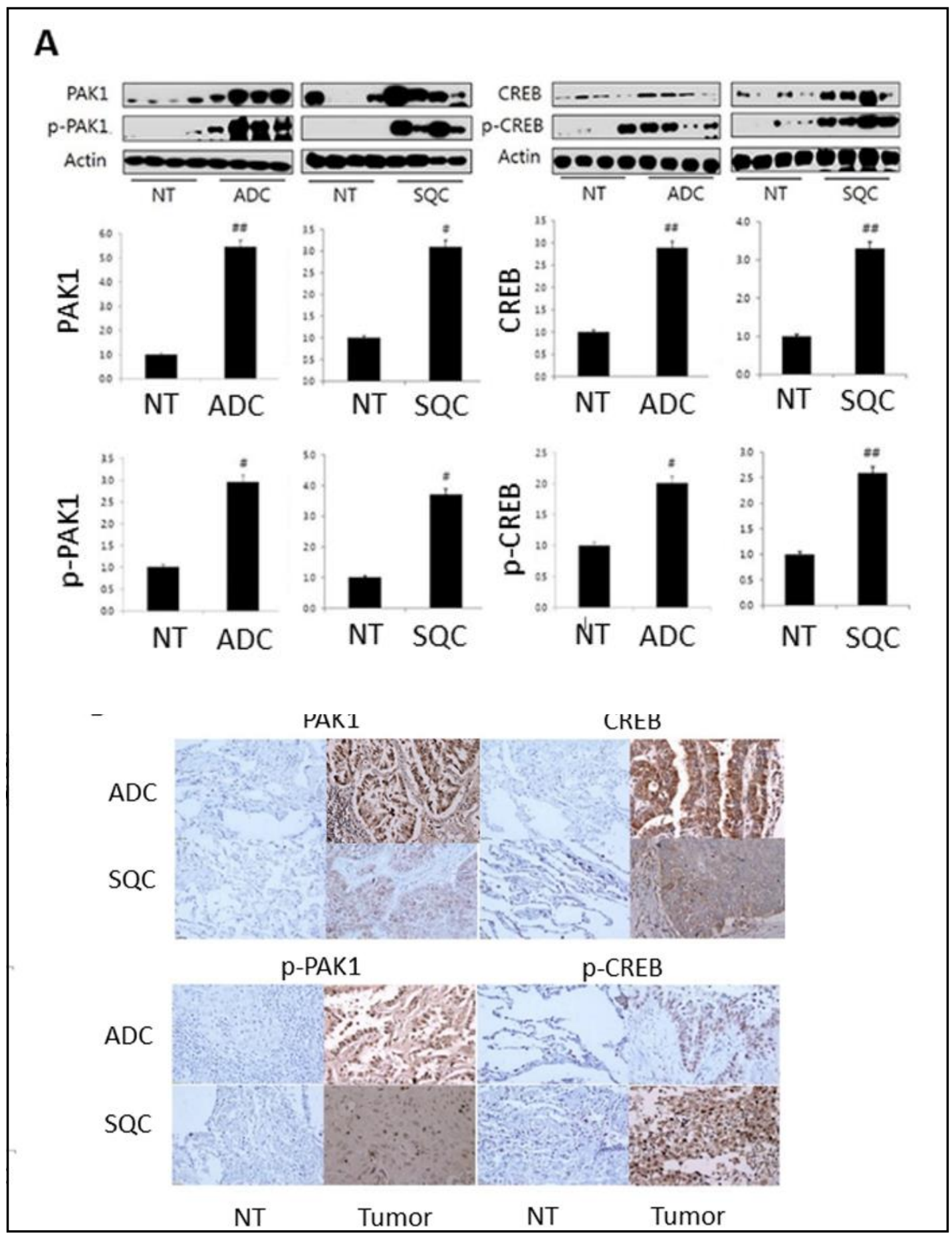

Fig. 2. Production of PAK1, p-PAK1, CREB, and p-CREB in human NSCLC tissues and adjacent normal epithelial tissues. A, Western blot analysis of PAK1, p-PAK1, CREB, and p-CREB levels. Proteins obtained from eight adenocarcinoma (ADC) and eight squamous-cell carcinoma (SQC) tissue specimens and pairmatched normal-tissue (NT) specimens were examined by western blot. $\beta$-actin was detected as an internal control. The experiment was independently repeated three times, producing similar results. Values are shown as the mean $\pm \mathrm{SD}\left(\mathrm{n}=8\right.$ replicates). ${ }^{\#} \mathrm{P}<0.05$ vs. NT group; ${ }^{\# P} \mathrm{P}<0.01$ vs. NT group.B, Fixed NSCLCtissue and adjacent epithelial NT specimens were subjected to immunohistochemical staining and scored according to the criteria mentioned in the Materials and Methods section. Representative images (right) were captured at a magnification of $400 \mathrm{X}$.

\section{KARGER}


PI staining indicated that the percentage of apoptotic cells increased by $\sim 11 \%$ in PAK1-knockdown H1703 cells, relative to controls (Fig. 3C). After PAK1 siRNA transfection, we observed a significant increase in TUNEL-positive dots (Fig. 3D). As shown in Fig. 3E, PAK1-silenced cells also exhibited proteolytic degradation of PARP and elevations in the expression of cleaved caspase-3 and caspase-9. Moreover, the expression levels of the pro-apoptotic Bax protein were elevated, whereas levels of the anti-apoptotic Bcl-2 protein were unchanged in H1703 cells. These results suggested that PAK1 silencing induced cell cycle arrest and apoptosis in $\mathrm{H} 1703$ cells, and indicated that the consequences of PAK1 blockade involved marked anti-proliferative effects in squamous-cell NSCLC cells.

\section{Roles of PAK1 in tumor growth}

Having established that PAK1 influences carcinogenesis in squamous-cell carcinoma cells, we further examined its role in tumor growth in vivo by subcutaneously injecting control or PAK1-knockdown cells. We observed statistically significant suppression of tumor growth (tumor volume [mm3], mean \pm SD; control siRNA vs. PAK1 siRNA: 1, $096 \pm 467.707 \mathrm{~mm} 3$ vs. $502.301 \pm 286.702 \mathrm{~mm} 3$ on day 28 , respectively; 2-sided t test, \#P $<0.05$; Fig. $4 \mathrm{~A}$ and B) and xenograft masses derived from mice receiving PAK1-knockdown cells, as compared with control cells (tumor volume [mg], mean \pm SD; control siRNA vs. PAK1 siRNA: $273.529 \pm$ $15.873 \mathrm{mg}$ vs. $12.414 \pm 21.578 \mathrm{mg}$ on day 28 , respectively; 2 -sided t test, \#P < 0.05; Fig. 4C). To demonstrate silencing of PAK1, immunohistochemistry analysis was performed to show the level of PAK1 from PAK1 siRNA tumors harvested at the end of experiments (Fig. 4D). These results indicated that PAK1 is an important component in tumor growth of squamouscell lung cancer.

\section{Regulation of CREB by PAK1 in H1703 cells}

We then explored the mechanism underlying the PAK1-mediated promotion of cell proliferation. Interestingly, correlation analysis by western blotting showed possible associations between PAK1 and p-CREB levels ( $n=16, r=0.69, P=0.018$; Fig. 5A), suggesting that PAK1 might phosphorylate CREB in squamous-cell lung cancer.

We then performed a western blot analysis to confirm the relationship between PAK1 and p-CREB, finding that CREB phosphorylation was significantly downregulated following the transfection of H1703 cells with PAK1 siRNA (2-fold, ${ }^{\# \#} \mathrm{P}<0.001$; Fig. 5B), although the total CREB protein levels were unchanged $(\mathrm{P}=0.4$; Fig. $5 \mathrm{~B})$. Consistent with this result, PAK1 overexpression following transfection with the pCMV6M-Pak1 L107F plasmid upregulated CREB phosphorylation at Ser133 in $\mathrm{H} 1703$ cells (Fig. 5C). These results demonstrated that at least a part of PAK1 phosphorylates CREB at Ser133. Until now, the major focus of the study has been on the cytosolic functions of PAK1, where it is a significant modulator of cytoskeletal reorganization, consequently playing an important role in cell survival, migration, and invasion. Our immunofluorescence assay showed that PAK1 mostly localized in the cytosol in serum-free medium, which has no growth factors. However, serum stimulation-containing growth factors resulted in the intracellular localization of PAK1 and CREB in the nucleus (Fig. 5D). We further studied PAK1 bound to CREB by co-immunoprecipitating PAK1 with an anti-CREB antibody in PAK1-overexpressing H1703 cells, finding that PAK1 complexes with CREB in the nucleus after serum stimulation (Fig. 5E). 

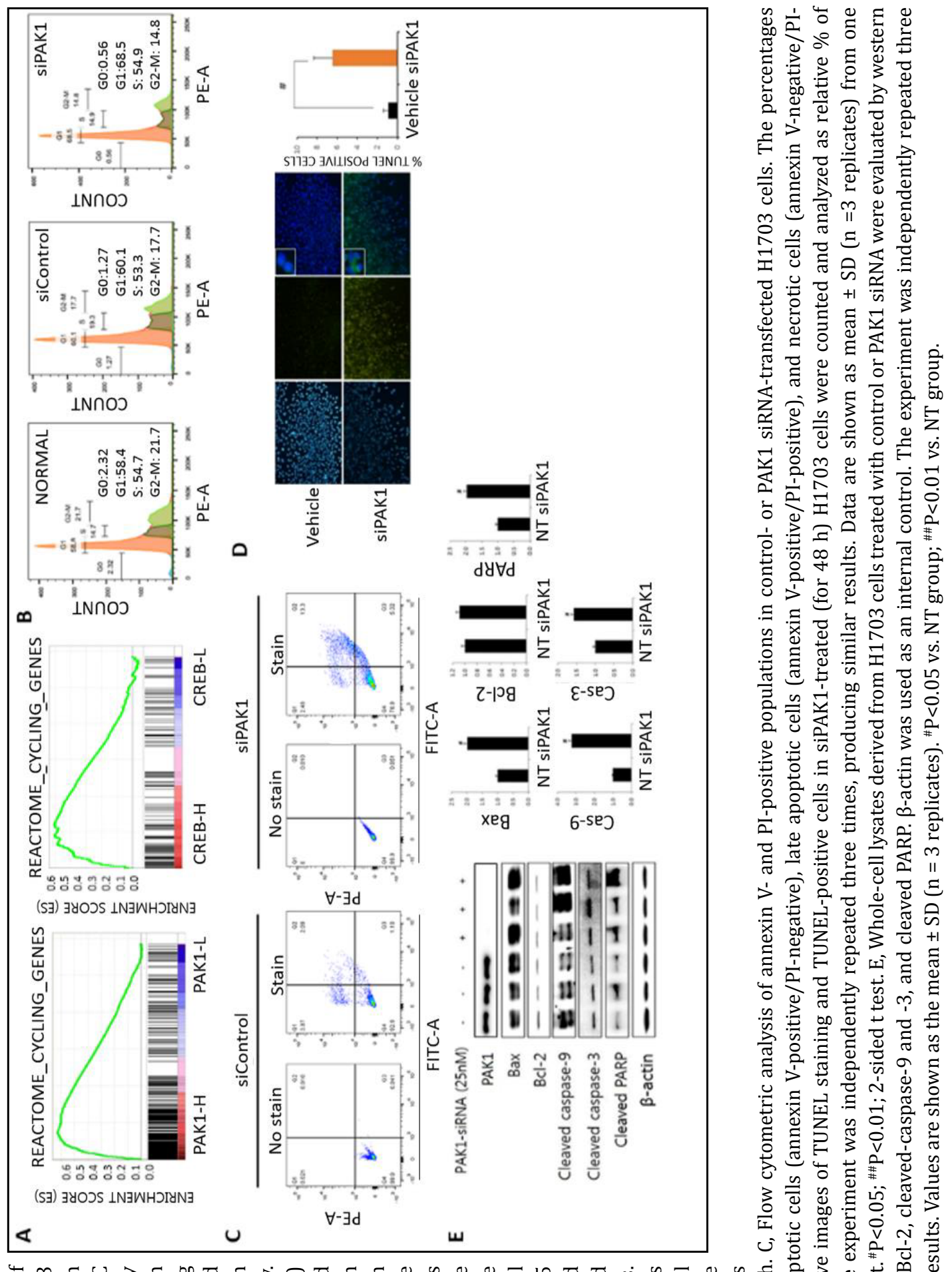

ัั

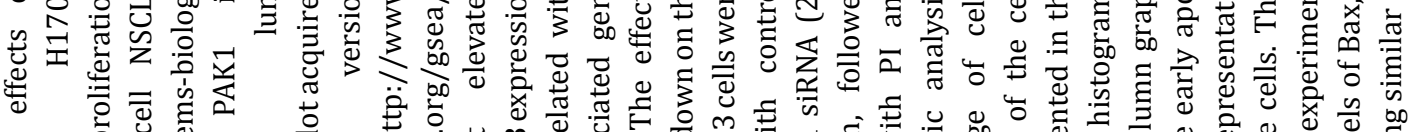

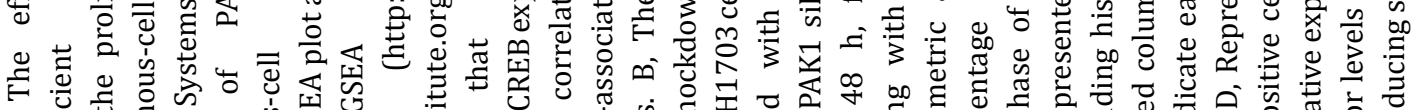

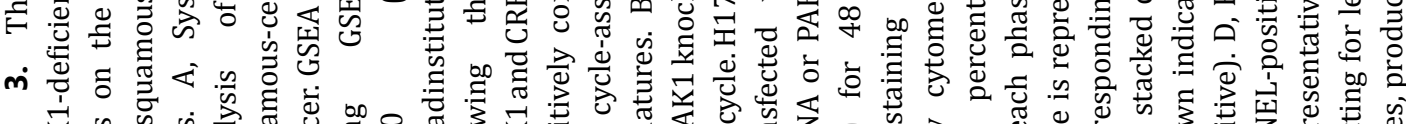

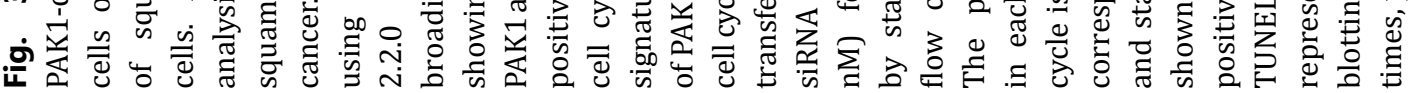
KARGER 
A
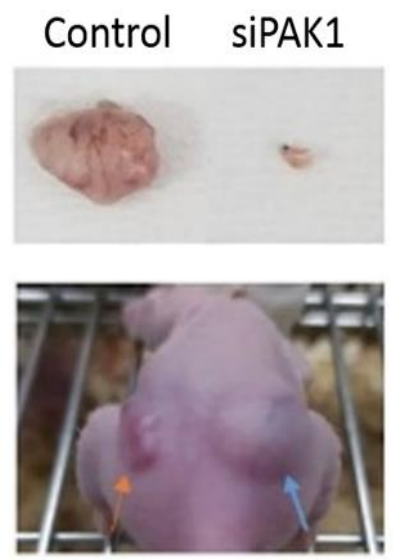

C

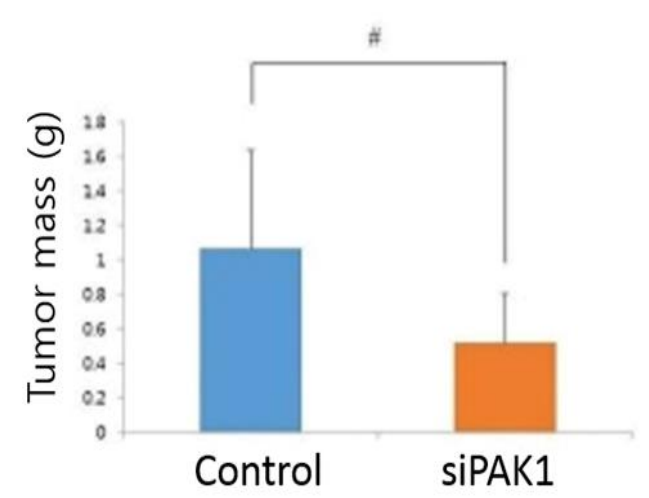

D
B
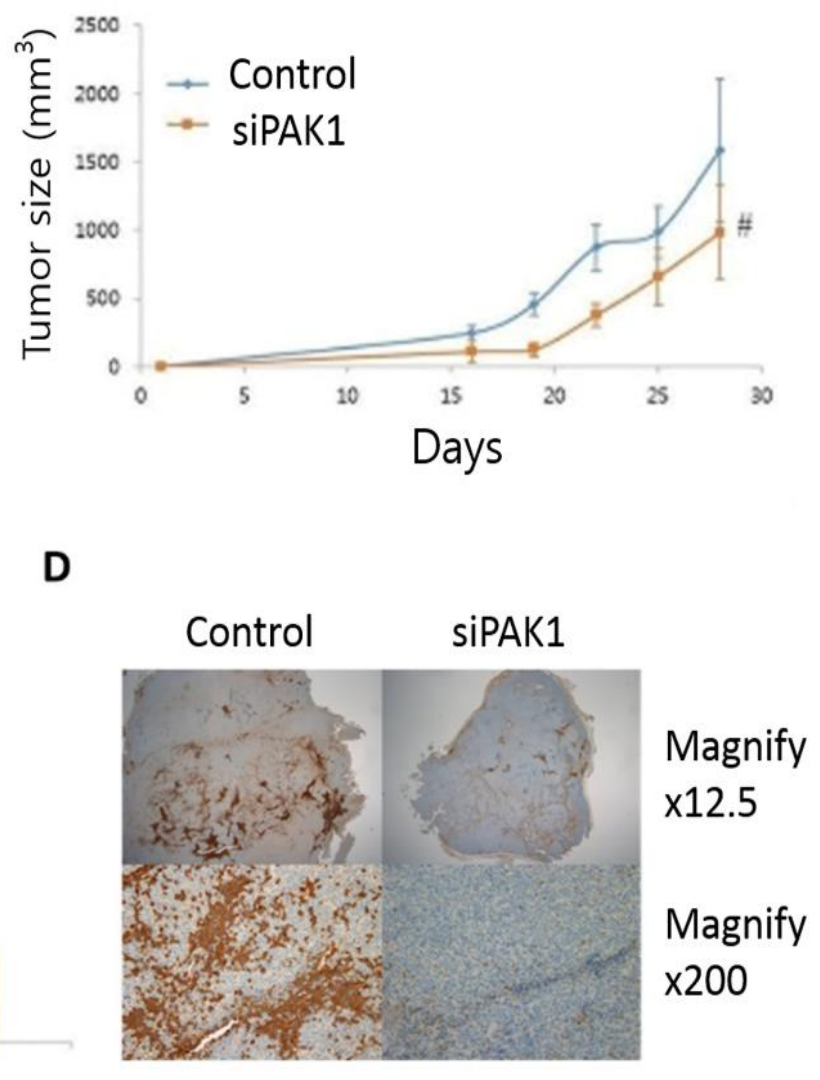

Fig. 4. The effects of PAK1-knockdown on tumor growth in vivo. A, Representative images of xenografts derived from the transfer of control- or PAK1 siRNA-treated H1703 cells. After a 48-h siRNA transfection (25 nM), cells were injected subcutaneously into the right and left side flanks of female BALB/c-nude mice (xenografts: $n=10$ /group). B, Tumor volumes and $C$, xenograft masses resulting from control- or PAK1 siRNA-transfected H1703 cells were assessed. Tumor volumes were measured with digital calipers and calculated according to the formula: $0.52 \times$ length $\times$ width $^{2}$, and tumor masses were evaluated at 4-weeks post-injection. D. Representative images of PAK1 levels from PAK1 siRNA tumors harvested at the end of experiments. The experiment was independently repeated three times, producing similar results. Value are shown as the mean $\pm \mathrm{SD}(\mathrm{n}=10$ replicates $) .{ }^{\#} \mathrm{P}<0.05 ;{ }^{\# \#} \mathrm{P}<0.01 ; 2$-sided t test.

\section{Discussion}

PAK1 has been implicated in the tumorigenesis of several types of cancer, including hormone-dependent breast and ovarian cancers, gastrointestinal cancers, and central nervous system cancers $[2,16]$. In this study, we discovered the role of the PAK1-CREB axis in the development and pathogenesis of squamous-cell NSCLC. Our results showed that PAK1 and CREB expression levels were significantly elevated in patients with squamouscell carcinoma in their lungs and that the phosphorylated forms of both proteins were also upregulated in parallel. Ong et al. [9] reported that elevated PAK1 expression is prevalent in $64 \%$ of squamous-cell NSCLCs. Our data were consistent with these findings, as NSCLC tissues showed higher levels of both PAK1 and p-PAK1, compared with the corresponding 


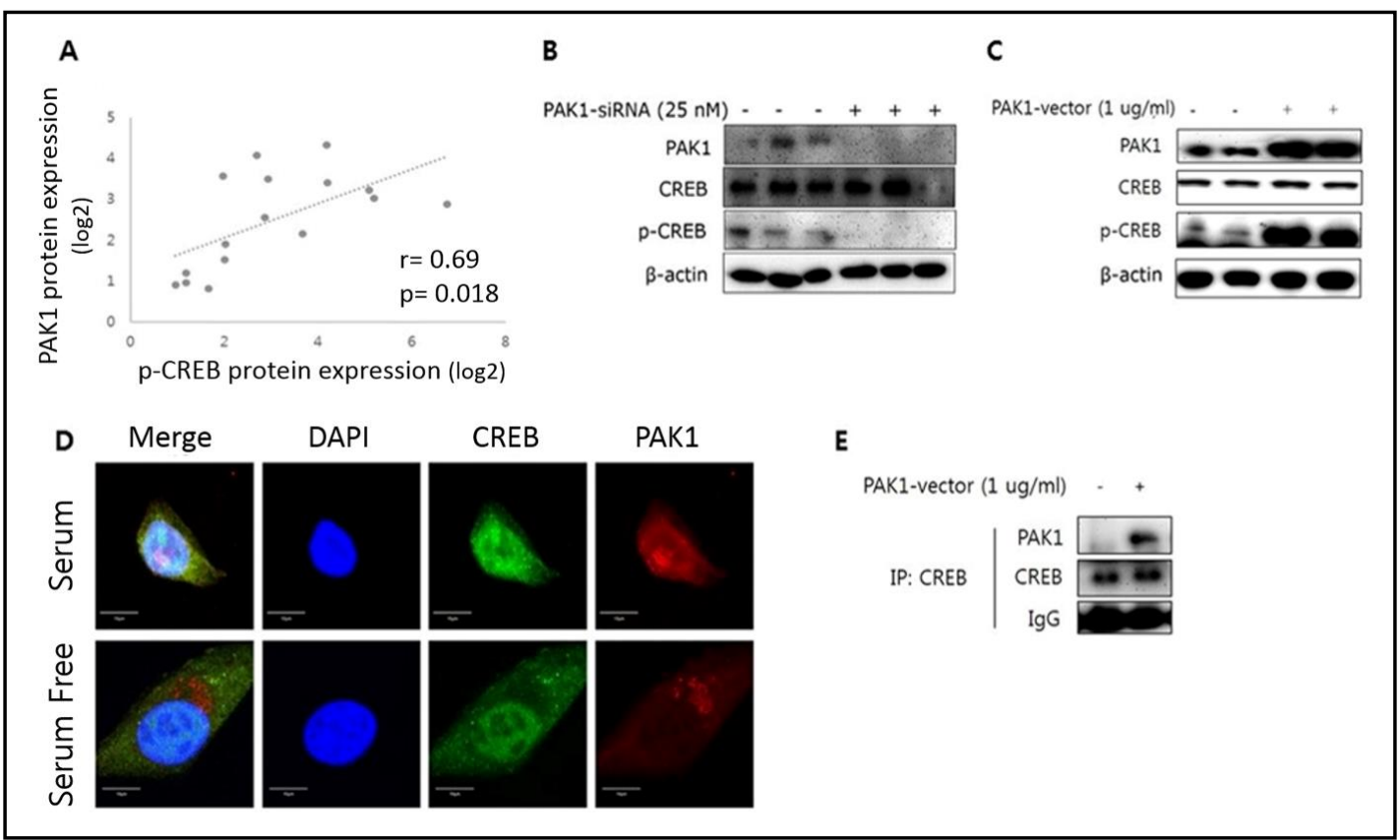

Fig. 5. The regulation of CREB by PAK1 in H1703 cells. A, Correlations between PAK1 and CREB protein levels based on immunohistochemical scoring $(n=16)(r=0.698 ; P=0.018)$. B, CREB and p-CREB levels following transfection of H1703 cells with PAK1 siRNA. C, Transfection of H1703 cells with an empty vector or a PAK1-overexpression plasmid (pCMV6M-Pak1 L107F). D, Immunofluorescence confocal assays showed that PAK1 redistributes into the nucleus and colocalizes (red) with CREB (green) after serum stimulation of H1703 cells (x3000). E, Aliquots of cell lysates were co-immunoprecipitated using an anti-CREB and an anti-PAK1 antibody. The experiment was independently repeated three times, producing similar results. Data are shown as mean $\pm \mathrm{SD}\left(\mathrm{n}=3\right.$ replicates) from one representative experiment. ${ }^{\#} \mathrm{P}<0.05$; ${ }^{\# \#} \mathrm{P}<0.01$; 2-sided t test.

expression levels in paired normal-tissue specimens (Fig. 2). Immunohistochemical analysis of whole NSCLC-tissue sections and adjacent, normal epithelial-tissue specimens confirmed that significantly higher PAK1 and p-PAK1 production occurred in tumor specimens, relative to normal tissues. Interestingly, our data also showed nuclear expression of PAK1 (62.5\%) and p-PAK1 (75.0\%) in squamous-cell carcinomas (Table 3). The biological function of PAK1 in the nucleus remained poorly understood until now; however, Lightcap et al. [17] reported that nuclear import of PAK1 is indispensable for vertebral development. Li et al. [18] also suggested that the nuclear functions of PAK1 are important to ensure the completion of mitosis. Here, we showed that during nuclear transport of PAK1 in serum containing growth factors, PAK1 complexes with CREB (Fig. 5D, E).

The results of our immunohistochemical study on squamous NSCLC TMAs, obtained from 60 patients, showed that increased PAK and p-PAK1 levels were associated with a trend toward decreased overall survival durations, although the log-rank test of both curves showed no significant difference. Nonetheless, the estimation of overall survival durations related to each clinicopathological characteristic of NSCLC patients showed that PAK1 expression positively correlated with $\mathrm{N}$ stage $(\mathrm{P}=0.03$ ) and TNM staging (III; $\mathrm{P}=0.04$ ). The observed effects of increased PAK1 and p-PAK1 levels on clinicopathological parameters suggests that PAK1 is a useful biomarker for predicting survival duration and metastasis; therefore, we believe that this issue warrants further research to understand the correlation between PAK1 and the risk of squamous NSCLC.

Our in vitro results indicated that $P A K 1$ silencing induced cell cycle arrest and apoptosis in squamous-cell carcinoma cells, as verified by flow cytometric analysis, TUNEL assays, and detection of the signature cleavages of PARP and caspases (Fig. 3B-E). Consistent with these results showing PAK1-mediated regulation of squamous-cell lung-cancer cell proliferation, 
xenograft models showed that PAK1 silencing reduced the size of lung-cancer tumors (Fig. 4). Therefore, our findings suggested that PAK1 plays a critical role in the proliferation of squamous-cell carcinomas.

Several different downstream pathways have been shown to mediate oncogenic signaling related to PAK1 $[19,20]$, but it is not clear which pathways regulate the progression of lung cancer. Recently, PAK4, a group-II PAK family member, was shown to promote prostate cancer progression through CREB [10]. Thus, we investigated whether the PAK1-CREB axis plays a pathogenic role in squamous lung cancers. Correlation analysis indicated that PAK1 was positively correlated with $\mathrm{p}$-CREB levels $(\mathrm{r}=0.69, \mathrm{P}<0.001$; Fig. $5 \mathrm{~A})$. Subsequent western blot analysis showed that CREB phosphorylation at Ser133 was significantly decreased following PAK1 siRNA transfection in H1703 cells $(\mathrm{P}<0.005)$, although CREB protein levels were not reduced $(\mathrm{P}=0.40$; Fig. $5 \mathrm{~B})$. Plasmid-based PAK1 overexpression resulted in CREB phosphorylation at Ser133. In agreement, several lines of evidence show that CREB activates the transcription of target genes in response to various stimuli, including protein kinase $\mathrm{A}$, pp90 ribosomal S6 kinase, and $\mathrm{Ca}^{2+} /$ calmodulin-dependent protein kinases. These kinases all phosphorylate CREB at Ser133, which is required for CREB-mediated transcription $[21,22]$. Therefore, phosphorylation of CREB at Ser133 by PAK1 might be, at least in part, involved in CREB-mediated transcription.

\section{Conclusion}

In summary, the present study provides important initial insights into the role of the PAK1-CREB axis in the development and pathogenesis of NSCLC. First, PAK1 and CREB were found to be overexpressed in banked squamous NSCLC specimens. Second, modulation of PAK1 expression levels independently correlated with decreased overall survival durations, $\mathrm{N}$ stage, and TNM stage. PAK1 deficiency also correlated experimentally with cell cycle arrest and apoptosis in vitro and tumor growth in vivo. Third, PAK1 phosphorylated CREB at Ser133 and complexed with CREB in squamous NSCLC samples. To the best of our knowledge, this represents the first study providing evidence that PAK1 modulates CREB phosphorylation in squamous-cell NSCLC. Because squamous-cell histological subtypes offer limited therapeutic approaches, our mechanistic findings on the role of the PAK1-CREB axis in the carcinogenesis of squamous-cell carcinomas might provide important implications in developing therapeutic strategies and targeted agents for treating squamous-cell lung cancer.

\section{Acknowledgements}

The authors would like to thank Dr. Chernoff J (Fox Chase Cancer Center, University of Pennsylvania) for a kind gift of the PAK1 constructs for this study. The biospecimens and data used for this study were provided by the Biobank of Pusan National University Hospital, a member of the Korea Biobank Network and of Pusan National University Yangsan Hospital. We also thank the Aging Tissue Bank (Busan, Korea) for supplying research materials. This work was supported by the Research Institute for Convergence of Biomedical Science and Technology, Pusan National University Yangsan Hospital grant (04-2017-004; S.H. Yoon) and the National Research Foundation of Korea (NRF) grant funded by the Korea government (MSIT) (NRF-2018R1C1B6004250).

\section{Disclosure Statement}

The authors declare that no conflict of interests exist. 


\section{Cellular Physiology Cell Physiol Biochem 2018;50:304-316 \begin{tabular}{ll|l} 
and Biochemistry Published onlIne: 4 October 2018 & $\begin{array}{l}\text { (c) } 2018 \text { The Author(s). Published by S. Karger AG, Basel } \\
\text { www.karger.com/cpb }\end{array}$
\end{tabular}}

Chung et al.: PAK1/CREB Axis in Squamous NSCLC

\section{References}

$>1$ Eswaran J, Li DQ, Shah A, Kumar R: Molecular pathways: targeting p21- activated kinase 1 signaling in cancer--opportunities, challenges, and limitations. Clin Cancer Res 2012;18:3743-3749. Kumar R, Gururaj AE, Barnes CJ: p21-activated kinases in cancer. Nat Rev Cancer 2006;6:459-471. Yeo D, He H, Patel O, Lowy AM, Baldwin GS, Nikfarjam M: FRAX597, a PAK1 inhibitor, synergistically reduces pancreatic cancer growth when combined with gemcitabine. BMC Cancer 2016;20:16-24.

4 Wang Z, Jia G, Li Y, Liu J, Luo J, Zhang J, Xu G, Chen G: Clinicopathological signature of p21-activated kinase 1 in prostate cancer and its regulation of proliferation and autophagy via the mTOR signaling pathway. Oncotarget 2017;8:22563-22580.

55 Oladimeji P, Skerl R, Rusch C, Diakonova M: Synergistic activation of ER $\alpha$ by estrogen and prolactin in breast cancer cells requires tyrosyl phosphorylation of PAK1.Cancer Res 2016;76:2600-2611.

-6 Kim E, Youn H, Kwon T, Son B, Kang J, Yang HJ, Seong KM, Kim W, Youn B: PAK1 tyrosine phosphorylation is required to induce epithelial-mesenchymal transition and radioresistance in lung cancer cells. Cancer Res 2014;74:5520-5531.

7 Wu D, Wu TC, Chen CY, Lee H: PAK1 is a novel therapeutic target in tyrosine kinase inhibitor-resistant lung adenocarcinoma activated by PI3K/AKT signaling regardless of EGFR mutation. Clin Cancer Res 2016;22:5370-5382.

8 Chen MJ, Wu DW, Wang YC, Chen CY, Lee H: PAK1 confers chemoresistance and poor outcome in non-small cell lung cancer via $\beta$-catenin-mediated stemness. Sci Rep 2016;6:34933.

-9 Ong CC, Jubb AM, Haverty PM, Zhou W, Tran V, Truong T, Turley H, O’Brien T, Vucic D, Harris AL, Belvin M, Friedman LS, Blackwood EM, Koeppen H, Hoeflich KP: Targeting p21-activated kinase 1 (PAK1) to induce apoptosis of tumor cells. Proc Natl Acad Sci U S A 2011;108:7177-7182.

-10 Park MH, Lee HS, Lee CS, You ST, Kim DJ, Park BH, Kang MJ, Heo WD, Shin EY, Schwartz MA, Kim EG: p21Activated kinase 4 promotes prostate cancer progression through CREB. Oncogene 2013;32:2475-2482.

$>11$ Conkright MD, Montminy M: CREB: the unindicted cancer co-conspirator. Trends Cell Biol 2005;15:457459.

12 Shankar DB, Sakamoto KM: The role of cyclic AMP binding protein (CREB) in leukemia cell proliferation and acute leukemias. Leuk Lymphoma 2004;45:265-270.

13 Seo HS, Liu DD, Bekele BN, Kim MK, Pisters K, Lippman SM, Wistuba II, Koo JS: Cyclic AMP response element-binding protein overexpression: a feature associated with negative prognosis in never smokers with non-small cell lung cancer. Cancer Res 2008;68:6065-6073.

14 Aggarwal S, Kim SW, Ryu SH, Chung WC, Koo JS: Growth suppression of lung cancer cells by targeting cyclic AMP response element-binding protein. Cancer Res 2008;68:981-988.

15 Kim DH, Chung JH, Yoon JS, Ha YM, Bae S, Lee EK, Jung KJ, Kim MS, Kim YJ, Kim MK, Chung HY: Ginsenoside Rd inhibits the expressions of iNOS and COX-2 by suppressing NF- $\mathrm{BB}$ in LPS-stimulated RAW264.7 cells and mouse liver. J Ginseng Res 2013;37:54-63.

-16 Dummler B, Ohshiro K, Kumar R, Field J: Pak protein kinases and their role in cancer. Cancer Metastasis Rev 2009;28:51-63.

17 Lightcap CM, Kari G, Arias-Romero LE, Chernoff J, Rodeck U, Williams JC: Interaction with LC8 is required for Pak1 nuclear import and is indispensable for zebrafish development. PLoS One 2009;4:e6025.

-18 Li F, Adam L, Vadlamudi RK, Zhou H, Sen S, Chernoff J, Mandal M, Kumar R. p21- activated kinase 1 interacts with and phosphorylates histone H3 in breast cancer cells. EMBO Rep 2002;3:767-773.

19 Molli PR, Li DQ, Murray BW, Rayala SK, Kumar R. PAK signaling in oncogenesis. Oncogene 2009;28:25452555.

20 Qing H, Gong W, Che Y, Wang X, Peng L, Liang Y, Wang W, Deng Q, Zhang H, Jiang B: PAK1-dependent MAPK pathway activation is required for colorectal cancer cell proliferation. Tumour Biol 2012;33:985-994.

21 Shawitz AJ, Greenberg ME: CREB: a stimulus-induced transcription factor activated by a diverse array of extracellular signals. Annu Rev Biochem 1999;68:821-861.

22 West AE, Chen WG, Dalva MB, Dolmetsch RE, Kornhauser JM, Shaywitz AJ, Takasu MA, Tao X, Greenberg ME: Calcium regulation of neuronal gene expression. Proc Natl Acad Sci U S A 2001;98:11024-11031. 